\title{
Single crystal-like performance in solution-coated thin-film organic field-effect transistors
}

Freddy G. del Pozo, ${ }^{1}$ Simone Fabiano, Raphael Pfattner, Stamatis Georgakopoulos, Sergi Galindo, Xianjie Liu, Slawomir Braun, Mats Fahlman, Jaume Veciana, Concepció Rovira, Xavier Crispin, Magnus Berggren,* Marta Mas-Torrent*

Dr. F. G. del Pozo, Dr. R. Pfattner, Dr. S. Georgakopoulos, S. Galindo, Prof. J. Veciana, Prof. C. Rovira, Dr. M. Mas-Torrent

Institut de Ciència de Materials de Barcelona (ICMAB-CSIC) and Networking Research Center on Bioengineering, Biomaterials and Nanomedicine (CIBER-BBN), Campus de la UAB, 08193,

Bellaterra, Spain.

E-mail: mmas@icmab.es

Dr. S. Fabiano, Dr. X. Crispin, Prof. M. Berggren.

Laboratory of Organic Electronics, Department of Science and Technology, Linköping University, SE-601 74, Norrköping, Sweden.

E-mail: magnus.berggren@liu.se

Dr. X. Liu, Dr. S. Braun, Prof. M. Fahlman

Department of Physics, Chemistry and Biology, Linköping University SE-581 83, Linköping, Sweden.

${ }^{1}$ Current address: Universidad Técnica de Ambato (Ecuador)

Keywords: Organic field-effect transistors, thin-film coating, charge carrier mobility, temperature-independent transport, device stability

In electronics, the field-effect transistor (FET) is a crucial corner stone and successful integration of this semiconductor device into circuit applications requires stable and ideal electrical characteristics over a wide range of temperatures and environments. Solution processing, using printing or coating techniques, has been explored to manufacture organic field-effect transistors (OFET) on flexible carriers; enabling radically novel electronics applications. Ideal electrical characteristics, in organic materials, are typically only found in single crystals. Tiresome growth and manipulation of these hamper practical production of flexible OFETs circuits. To date, neither devices nor any circuits, based on solution-processed 
OFETs, has exhibited an ideal set of characteristics similar or better than today's FET technology based on amorphous silicon (a-Si FETs). Here, we report bar-assisted meniscus shearing of dibenzo-tetrathiafulvalene to coat-process self-organized crystalline organic semiconducting domains with high reproducibility. Including these coatings as the channel in OFETs, we observe electric field- and temperature-independent charge carrier mobility and no bias stress effects. Further, we measure record-high gain in OFET inverters and exceptional operational stability in both air and water.

\section{Introduction}

Organic electronics have undergone significant progress over the past 20 years and prototypes for a variety of applications have begun to emerge including sensors, memory elements, and disposable electronics. The best performing electronic devices to date are those comprising single crystals of organic semiconductors. ${ }^{[1,2]}$ However, due to their poor mechanical properties, single crystals are neither suitable for large-area applications nor compatible with fast high-throughput industrial-scale fabrication. Therefore, to make organic electronics competitive with the inorganic counterparts and to promote a competitive market entry, the fabrication process should rely on very simple and inexpensive approaches, in which the deposition of the organic semiconductors should ideally be performed from solution in ambient conditions. ${ }^{[3,4]}$ During the last years, significant progresses have been made toward the development of viable printed electronics based on solution-processed organic field-effect transistors (OFETs). ${ }^{[5-7]}$ The general guidelines for the requirements of OFETs are: low processing temperature $\left(<120^{\circ} \mathrm{C}\right),{ }^{[8]}$ high on/off ratio $\left(>10^{6}\right),{ }^{[9]}$ high reproducibility, ${ }^{[10]}$ high uniformity ${ }^{[11]}$ and high stability (temperature, ${ }^{[12]}$ bias stress ${ }^{[13]}$ and lifetime $\left.{ }^{[14]}\right)$. However, all these requirements do not typically coexist in printed OFETs, thus hampering their further exploitation and success in large-area electronics applications. 
Because of the weak van der Waals interaction, between small organic molecules, the morphology and molecular packing of solution-processed OFET films are crucial to their electrical performance. ${ }^{[15]}$ Yet, obtaining defect-free semiconducting films directly through solution-coating remains challenging, due to the out-of-equilibrium deposition conditions (i.e. fast solvent evaporation, fluid flow instabilities, etc.). ${ }^{[16]}$ Hence, the task to combine the perfect materials and to obtain an optimized OFET with ideal electrical characteristics is certainly a demanding task. Although, today's printed OFET displays a charge carrier mobility comparable to that of benchmark thin-film amorphous silicon devices $(\mu \sim 0.5-1.0$ $\mathrm{cm}^{2} \mathrm{~V}^{-1} \mathrm{~s}^{-1}$ ), the solution processed OFETs suffer from low reproducibility of the high charge carrier mobility, strongly activated charge transport, and high degradation rate (i.e. bias stress effects). ${ }^{[17]}$ These shortcomings, combined with the limiting air-instability typically observed for organic semiconductors, make still unclear whether solution deposition will offer sufficient technological and commercial advantages over the existing inorganic technologies.

We report the formation of solution-processable, highly crystalline thin films by a simple bar-assisted meniscus shearing method (BAMS) in ambient conditions using a blend solution of dibenzo-tetrathiafulvalene (DB-TTF) and polystyrene (PS). This process results, in one step, to the passivation of the dielectric surface and simultaneously the formation of a continuous self-encapsulated polycrystalline organic semiconductor thin film. The devices exhibit electrical characteristics matching those typically reported for devices comprising single crystals of the same material as well as those for a-Si FETs and they show, additionally, excellent operational stability in air and water and record-high gain in inverters. A temperature and field-independent device mobility with remarkably low trap density of states is observed, suggesting high crystalline quality of our solution-processed semiconducting films.

\section{Results and Discussion}


DB-TTF (Figure 1) is readily available and was selected as the active semiconductor because it is soluble in common organic solvents, thus avoiding the necessity of using precursors or other solubilizing routes. Furthermore, it has been shown to be a promising organic semiconductor due to its electronic and supramolecular structure. Hole mobility in the range $0.1-1 \mathrm{~cm}^{2} /$ Vs has been reported for solution-processed single crystals of DB-TTF, ${ }^{[18,19]}$ whereas devices comprising vacuum-deposited DB-TTF thin films show field-effect mobility that ranges from $10^{-2}$ to $10^{-1} \mathrm{~cm}^{2} /$ Vs (Figure S1). ${ }^{[20-23]}$ However, typically the devices are reported to exhibit large threshold voltages $\left(\mathrm{V}_{\mathrm{TH}}>20 \mathrm{~V}\right)$ and are very unstable in environmental conditions

We proceed to prepare films based on blends of DB-TTF with polystyrene with $\mathrm{M}_{\mathrm{w}}=$ $3000 \mathrm{~g} / \mathrm{mol}$ (PS). Solutions with different DB-TTF/PS ratios (i.e., 1:1, 2:3, 1:2 and 1:3) in 2 weight-\% in chlorobenzene were tested. After this optimization process, the best results were found using the blends of DB-TTF/PS 1:2. A bar coater was employed to cast the films, in which a smooth cylindrical bar was positioned approx. $300 \mu \mathrm{m}$ above cleaned $\mathrm{Si} / \mathrm{SiO}_{2}$ substrates with pre-fabricated gold electrodes that was heated to $105{ }^{\circ} \mathrm{C} .50 \mu \mathrm{L}$ of the blend solution was deposited between the bar and the substrate forming a confined meniscus. Immediately, the solution was sheared at a speed of approx. $1 \mathrm{~cm} / \mathrm{s}$. As the bar moves, the meniscus is displaced and by convective self-assembly (i.e., evaporation-induced selfassembly) $^{[24-26]}$ a thin film is formed. In Fig. 1a this process is schematically illustrated. Notably, the complete experimental procedure was carried out under ambient conditions. After solution casting, the substrates were left under vacuum at $60^{\circ} \mathrm{C}$ for 2 hours in order to remove solvent traces. It should be noted that the BAMS coating of pure DB-TTF gives rise to the formation of non-homogenous films formed by long crystals not connected between them. Further, the use of the spin coating technique to form films using these blends solutions 
does not give rise to working devices due to the fact that in these conditions DB-TTF does not crystallize.

The films were characterized by polarized optical microscopy. They reveal high and long-range uniformity with large crystalline domains with grain sizes of about $180 \times 180 \mu \mathrm{m}$ (Fig. 1a, S2). Using Atomic Force Microscopy (AFM) we estimated that the surface roughness of the film (rms) was only $3.8 \mathrm{~nm}$ (Fig. S3). This value is considerably lower as compared to the vacuum sublimed film of DB-TTF (rms approx. $13.9 \mathrm{~nm}$, Fig. S4) and of one order of magnitude lower than some of the values previously reported for films of other small molecule-polymer blends, where a relatively larger roughness was attributed to the segregation and crystallization of the molecules at the film/air interface. ${ }^{[27-29]}$ In our case, the smoothness of the film is indicative that the DB-TTF crystals are embedded in the polymeric matrix. The total thickness of the DB-TTF/PS film was estimated by AFM to be around $29 \pm 4$ nm (Fig. S5). A high water contact angle is measured along the DB-TTF/PS film surfaces, a value that basically is equivalent to the one found for neat PS films. This suggests that the highly hydrophobic PS layer is capping the DB-TTF crystals in the self-organized blend system (Fig. S6). X-ray diffraction experiments were also performed on both the evaporated thin films and on the solution-processed blend films. DB-TTF is known to exhibit a complex polymorphism scenario, where four polymorphs have already been identified. ${ }^{[30-32]}$ Previous works reported single crystal OFETs based on the most favorable thermodynamically $\alpha$-phase, while on thin films the kinetically more favorable $\gamma$-phase is usually found. Accordingly, here also in both the evaporated and solution casted films, it was observed that the crystals formed belong to such $\gamma$-phase (Fig.1b). In the present context, information of a possible vertical phase separation is of great interest, since this would influence the formation of the semiconducting layer and then affect the contact resistance. This was investigated by analyzing the distribution of DB-TTF at different film depths using time-of-flight secondary 
ion mass spectrometry (ToF-SIMS). Figure 1c shows the analysis of the $\mathrm{S}, \mathrm{C}$ and $\mathrm{SiO}_{2}$ contents gathered by a depth profile starting from the sample surface and finally reaching the $\mathrm{SiO}_{2}$ /organic blend interface. From this measurement, we find that within the first few nm depth there is a significant diminishment in S but not in C, which points towards a decrease of DB-TTF from the top of the surface to the semiconductor/dielectric interface. X-ray photoelectron spectroscopy measurements have been performed to identify the chemical composition at the surface, more especially the ratio between the concentration of sulfur to carbon atoms. As shown in Fig.S7, the C1s and S2p core level lines, both measured at normal and grazing angle, are approximately of the same intensity, which suggests that the DBTTF/PS film is rather homogeneous within the surface area to a depth of a few nanometers. The analysis of the elemental composition gives a $\mathrm{C}$ vs. S ratio that equals 7.5 , which is more than twice the value obtained from the chemical formula of DB-TTF (3.5). PS is therefore present and do not exhibit any significant vertical segregation in the near-surface region. The ToF-SIMs and XPS data together with the results from the crossed polarizer/analyzer suggest that the top-surface of DB-TTF/PS film is composed of the segregated crystalline domains of DB-TTF, but they are embedded in a PS matrix.

The field-effect characteristics of the coated films are measured in air. Remarkably, these OFETs are stable in air, in stark contrast to transistors including evaporated DB-TTF. In Figure S1 it is shown that in one of the evaporated thin films prepared with $\mathrm{V}_{\mathrm{TH}}$ close to zero, a significant $\mathrm{V}_{\mathrm{TH}}$ shift is observed even when operated in a glove box (in darkness with $\left[\mathrm{O}_{2}\right]$ and $\left[\mathrm{H}_{2} \mathrm{O}\right]$ below 2 and 3 ppm, respectively). Moreover, when a DB-TTF OFET is taken out of the glove box, its performance is lost within a few seconds resulting, as is indicated by a strong increase of the off current, from dioxygen doping. Those first observations suggest that polystyrene has an active role in the observed stability of the DB-TFF/PS based transistors. Besides being extremely hydrophobic, polystyrene is also known to function as an efficient barrier to dioxygen and water with a permeability coefficient of $11 \mathrm{ml} \mathrm{cm}^{-2} \mathrm{~s}^{-1} \mathrm{~cm} \mathrm{mmHg}^{-1}$ (at 
$30{ }^{\circ} \mathrm{C}$ ) for $\mathrm{O}_{2}$ and $12000 \mathrm{ml} \mathrm{cm}^{-2} \mathrm{~s}^{-1} \mathrm{~cm} \mathrm{mmHg}^{-1}$ (at $25^{\circ} \mathrm{C}$ and $90 \% \mathrm{RH}$ ) for $\mathrm{H}_{2} \mathrm{O} .{ }^{\text {[33] }}$

Bottom-gate, bottom-contact devices using DB-TTF/PS as the active material give I-V characteristics that resembles an almost ideal fit to the gate voltage (or carrier density)independent model (Figure 2). ${ }^{[34]}$ Note that practically $100 \%$ of the prepared devices were fully functional, demonstrating a high reliability of the fabrication process that gives rise to very uniform films (Figure S8). In the transfer curve (Fig. 2a), the device turns on at a gate voltage $\mathrm{V}_{\mathrm{G}}=0 \mathrm{~V}$ and the channel current is enhanced by 6-7 orders of magnitude when the device is fully ON. No hysteresis is observed between the forward and reverse sweeps showing the absence of mobile species, which indicates a pristine semiconductor/dielectric interface. The transfer curves at high $\mathrm{V}_{\mathrm{G}}$ exhibit excellent linear behavior, implying a gatevoltage independent mobility and thus an efficient charge injection from the gold contact and negligible carrier trapping. The independency of mobility with the gate voltage is clearly elucidated in Fig. 2b and Fig. S9. A charge density-independent mobility has been observed mainly in devices based on single crystals. ${ }^{[35][36,37]}$ This important characteristic of singlecrystal OFETs contrasts sharply with the typical strong $\mathrm{V}_{\mathrm{G}}$-dependent mobility that is typically observed in OFETs. In the latter case, the density of localized states within the gap is so high that the Fermi level remains in the gap even at high $\mathrm{V}_{\mathrm{G}}$ values. In the output curve (Fig. S10), the drain current saturates at large drain voltages $\left(V_{D}\right)$, and the current-voltage relationship shows a linear behavior at low bias voltages. This corroborates the formation of ohmic contacts. Due to the ideal I-V characteristics, the mobility can be unambiguously estimated by taking first and second derivatives of the drain current for the linear and saturation regimes, respectively, giving average room-temperature mobility of approx. 0.2 $\mathrm{cm}^{2} \mathrm{~V}^{-1} \mathrm{~s}^{-1}$. (Fig. S11). The device performances are found to be independent of both channel length (Fig. S12) and orientation with respect to the casting direction (Supp. Info.), demonstrating that the charge transport in these films is fully isotropic. We also investigated 
the bias stress of DB-TTF/PS FETs when a fixed $V_{G}$ bias is applied to the devices during extended periods of time. It is known that a prolonged application of $\mathrm{V}_{\mathrm{G}}$ affects the currentvoltage characteristics of OFETs and is manifested mainly by a $\mathrm{V}_{\mathrm{TH}}$ shift, which stems from the entrapment of mobile charge carriers in localised electronic states. Fig. 2c shows the bias stress characteristics evaluated by applying a constant $V_{G}=-20 \mathrm{~V}$ and a $V_{D}=-2 \mathrm{~V}$, at the same time measuring the transfer characteristics over a time period of approx. 12 hours. No appreciable shift in the $\mathrm{V}_{\mathrm{TH}}$ is measured over this time. Taken all together, these results reveal the achievement of high performance DB-TTF/PS-based OFETs, and thus the availability of another organic semiconductor test bed for systematic charge transport studies. With such transistors, we achieved an environmentally stable depleted load inverters with gain larger than 300 at relatively low supply voltages $\left(V_{D D}=-30 \mathrm{~V}\right)$, being among the highest values ever reported for p-MOS inverters used in complex circuitry. ${ }^{[38]}$

The nature of the charge transport was investigated by measuring the current-voltage characteristics at different temperatures. Interestingly, a temperature independent mobility is observed over a wide range of temperatures spanning from room T down to $78 \mathrm{~K}$ (Figure 3a). It should be highlighted that such temperature-independent mobility dependency was reproducibly observed in different films. To connect the excellent DB-TTF/PS device performance with the intrinsic properties of the semiconducting material, we analyzed the trap density of states (trap DOS) of DB-TTF/PS-based OFETs. We applied the analytical method described in Ref. ${ }^{[39]}$ to the linear-regime-transfer characteristics of our OFETs. The charge per unit area induced in the OFET by the $V_{\mathrm{G}}$ bias is given by $Q=C V_{\mathrm{G}}$, where $C$ is the gate capacitance per area unit. The density of gap states $N(E)$ is given by $N(E)=$ $(C / q a)\left[1 /\left(d E / d V_{\mathrm{G}}\right)\right]$, where $q$ is the electronic charge, $a$ is the effective accumulation layer thickness and $E$ is the energy measured from the valence band edge. $E$ is approximated with the activation energy $E_{\mathrm{a}}\left(V_{\mathrm{G}}\right)$, which is defined by $\sigma\left(V_{\mathrm{G}}\right)=A \exp \left(-E_{\mathrm{a}} / k T\right)$, where $\sigma$ is the 
field-effect conductivity and $A$ is a constant. The activation energy is then determined from the measured data with a linear regression of $\ln \sigma$ vs $1 / T$ for each gate voltage according to ln $\sigma=\ln A-E_{\mathrm{a}} / k T$. An effective accumulation layer thickness of $a=7.5 \mathrm{~nm}$ is generally assumed. For our DB-TTF/PS-based FETs, we found almost constant field-effect conductivity between $78 \mathrm{~K}$ and $200 \mathrm{~K}$ and just a weak exponential activation above $200 \mathrm{~K}$. Thus, similarly to what has been reported for DB-TTF single crystals, ${ }^{[19]}$ this yields two datasets corresponding to a low temperature $(T<200 \mathrm{~K})$ and high temperature ( $T>200 \mathrm{~K})$ regime. Note that an ideal output characteristic showing no significant injection problems is observed even at low temperature ( $T=78 \mathrm{~K}$, Figure S13). Figure 3b gives $N(E)$ obtained from the smooth fit to the activation energy $E_{\mathrm{a}}\left(V_{\mathrm{G}}\right)$ with a trapping depth of $7.5 \mathrm{~nm}$. For the dataset at low temperature, the DOS close to the valence-band edge can be approximated by an exponential function falling rapidly by $2-3$ orders of magnitude within $20 \mathrm{meV}$, while for the dataset at $T>200 \mathrm{~K}$, the trap DOS decreases slowly to $\sim 10^{18} \mathrm{eV}^{-1} \mathrm{~cm}^{-3}$ at $0.3 \mathrm{eV}$. For comparison, representative density of gap states of DB-TTF single crystals ${ }^{[19]}$ is plotted together with the trap DOS of DB-TTF/PS thin films (Figure 3b). The latter shows a remarkably low trap DOS, which resembles that reported by Schmidt et al. ${ }^{[19]}$ for DB-TTF single crystals. Furthermore, although the method by Lang et al. leads to an underestimation of the slope of the trap DOS close to the valence-band edge, DB-TTF/PS thin films show a relatively lower trap DOS than pentacene thin films ${ }^{[40]}$ and are well within the range of benchmark single crystals of other organic semiconductors. ${ }^{[41]}$ This is remarkable because the trap densities in single crystal OFETs can be much lower than the trap densities in the best thin films transistors. ${ }^{[41]}$ Thus, this quantitative analysis relates the impressive device performance directly to the low trap DOS, which reveals the potential of DB-TTF as a high performance organic semiconductor.

The transport in organic semiconductors with high-crystallinity is typically described by the multiple trapping and release model $(\mathrm{MTR})^{[42,43]}$ that postulates that charge carriers 
flow in a delocalized band and are hindered by repeated trapping and thermal release events. As such, the mobility exhibits thermally activated behavior. Experiments in high-mobility single crystal OFETs, have exhibited features of band-like transport (i.e., carrier mobility increasing with lowering temperature) attributed mainly to the low concentration of traps at the organic semiconductor interface due to the employment of air-gap or low permittivity organic dielectrics and the high molecular order and absence of grain boundaries within the single crystals. ${ }^{[40,44-47]}$ Temperature independent mobility was reported for vacuum evaporated pentacene films on octadecyltrichlorosilane-treated $\mathrm{SiO}_{2}$ dielectric, ${ }^{[48]}$ while an apparent band-like transport has been observed for solution-processable pentacene derivatives and was attributed to localized transport limited by thermal lattice fluctuations rather than extendedstate conduction. ${ }^{[49]}$ However, the temperature independent-mobility profile observed in the present solution processed thin films completely prepared under environmental conditions has never been observed before. It should also be highlighted that such characteristics have been achieved with a non-air-stable organic semiconductor which additionally has not been purified by sublimation.

The shelf stability of the devices was also explored by measuring the devices for several weeks while storing them under ambient conditions. In Figure 4a, the evolution of the mobility and threshold voltage of a set of 10 devices measured 48 days after fabrication is plotted. All parameters can be considered to be very much constant along this time, revealing an excellent environmental stability of this material system. Hence, the reported fabrication technique has resulted in the self-encapsulation of DB-TTF in an inert polymer, permitting that a promising but highly unstable organic semiconductor can now be successfully operated in air.

Interestingly, our prepared blends reveal an excellent stability after being immersed in water, which is a highly uncommon capability of organic semiconductors since they typically degrade rapidly under humid conditions. ${ }^{[50-52]}$ A series of devices were dipped in mq-water 
for different time periods. After each immersion the device was blown with $\mathrm{N}_{2}$ to remove any water droplets, and subsequently, they were electrically characterized. Fig. 4b shows the semilog-transfer plots of one device measured after different water-immersion times up to 12 hours. The plots of the $\mu$ and $V_{T H}$, as function of immersion time, is given in figure $4 \mathrm{~b}$ (inset) and undoubtedly demonstrate the stability of the prepared devices, a feature which is very promising for the development of e.g. (bio)sensors.

With the unique combination of device parameters and properties, i.e. temperature and field independent mobility, air and water stability, no bias stress, uniformity of the coated films and reproducibility of the OFET performance, our tetrathiafulvalene-based OFETs offer sufficient technological and commercial advantages over existing a-Si FETs. Indeed, we routinely obtained saturated mobilities for our DB-TTF/PS-based FETs $\left(\sim 0.2 \mathrm{~cm}^{2} / \mathrm{Vs}\right)$ that are comparable to those of a-Si transistors. ${ }^{[53]}$ However, unlike a-Si our devices do not suffer from bias stress due to the very low trap DOS. In this respect, hydrogenated a-Si transistors are typically affected in fact by critical instability issues that limit their continuous operation. ${ }^{[54-56]}$ Such instabilities are due to a high density of deep traps, as suggested by the thermally activated transport that is typically observed for a-Si over a large range of $\mathrm{T}$ (from $77 \mathrm{~K}$ to $300 \mathrm{~K}$ same range as in the present study). ${ }^{[57,58]}$ This contrasts strongly with the temperature-independent mobility observed here for DB-TTF/PS-based OFETs (Fig. 4a). A direct consequence of such a low trap DOS in DB-TTF/PS is represented by the extraordinary high environmental stability of our OFETs. We believe that the capping of the hydroxyl groups with PS and the vertical phase separation that takes place in the blends achieving high crystallinity and excluding impurities, are responsible for the utterly low trap-DOS, which explains both the $\mathrm{V}_{\mathrm{G}^{-}}$and the temperature-independent. 


\section{Conclusions}

In conclusion, we have demonstrated the successful fabrication of high-performing OFETs, based on a polymeric blend including an unstable organic semiconductor that were manufactured using a solution shearing technique in ambient conditions. Through phase separation in the organic blend we find that polystyrene plays multiple roles. It acts as a promoting agent for the self-organization of the organic semiconductor during demixing, as an encapsulation material for the semiconductor and it also serves as a non-polar dielectric medium that ensures a low density of traps in the semiconductor channel. As a result, the electrical characteristics of the devices outperform those of a-Si FETs and meet the requirements for printing- and coating-manufacturing OFETs for several applications: 1) a high reproducibility of high performing devices, 2) air and water stability, and 3) long term environmental stability and basically no bias-stress effects. Outstandingly, the DB-TTF/PSblends show temperature-independent mobility pointing towards trap-free transport, which has never been reported for organic solution-processed thin films OFETs. Unipolar inverters exhibiting excellent gain values have been demonstrated as well. The combination of these device characteristics together with the low-cost of the materials and easy processing steps represents a significant step forward towards applications based on printed OFETs.

\section{Experimental Section}

Thermally evaporated thin-films of DB-TTF were prepared with a slow evaporation rate of about $0.5 \AA / \mathrm{s}$ on $\mathrm{Si} / \mathrm{SiO}_{2}$ as substrate and ITO/Au source and drain electrodes. The thickness of the evaporated organic film was found to be $d_{\text {film }}=110 \mathrm{~nm}$ extracted in AFM analysis.

Thin films of organic blends were produced using adapting a commercial wire coating equipment. DB-TTF and PS3000 were purchased from Sigma-Aldrich and used without further purification. Solutions of DB-TTF and PS3000 2\% wt in chlorobenzene were prepared 
by mixing solutions at a volume ratio of 1:2 DB-TTF:PS. The substrates employed for solution coating consisted of $\mathrm{Si} / \mathrm{SiO}_{\mathrm{x}}\left(200 \mathrm{~nm} \mathrm{SiO}_{\mathrm{x}}\right.$ ) from $\mathrm{Si}-$ mat with photolithography patterned electrodes of $4 \mathrm{~nm}$ of $\mathrm{Cr}$ and $40 \mathrm{~nm}$ of gold, deposited by thermal evaporation. The channel lengths vary from 20 to $200 \mu \mathrm{m}$ and channel widths lie in the $2 \mathrm{~mm}$ to $200 \mathrm{~mm}$ range. Substrates were cleaned by HPLC acetone and isopropanol and then dried under nitrogen. The organic semiconductor was deposited at ambient conditions using an RK control bar coater at speed around $1 \mathrm{~cm} / \mathrm{s}$ and keeping the substrates at $105{ }^{\circ} \mathrm{C}$. After deposition the coated substrates were dried in vacuum at $60^{\circ} \mathrm{C}$ for $2 \mathrm{~h}$ to remove solvent traces.

Electrical measurements were performed using a Keithley 2612A and home-made MATLAB software connected to the samples with a SUSS probe station, in darkness, at ambient conditions.

Optical microscope pictures were taken using an Olympus BX51 equipped with polarizer and analizer. Contact angle measurements were made using a DPA100 from Krüss and a $5 \mu \mathrm{l}$ mili-q water drop. Surface topography was examined by a 5500LS SPM system from Agilent Technologies and subsequent data analysis using Gwyddion software. Crystalline domains were quantified using ImageJ software. Chemical composition of the material was determined by Time-of-Flight Secondary Ion Mass Spectrometry (ToF-SIMS), surface sputter etching of the surface was accomplished with Cs+ beam, over a $300 \mu \mathrm{m} x$ $300 \mu \mathrm{m}$ area using $2 \mathrm{keV}$ and $1 \mathrm{keV}$ energy settings raster. A pulsed beam of $25 \mathrm{keV}$ Bi1 ions scanned over a $50 \mu \mathrm{m}$ x $50 \mu \mathrm{m}$ region centered within the sputtered area was used. Analysis cycle time was $100 \mu$ s and sputtering cycle was 1 s and 1000 ms flood gun compensation. A high current beam of low energy $(<20 \mathrm{eV})$ electrons was employed for charge compensation, and Negative ions were analyzed. X-ray diffraction measurements were carried out with a diffractometer from Rigaku, equipped with a rotating anode source. 
X-ray photoelectron spectroscopy measurements were carried out in an ultrahigh vacuum (UHV) surface analysis system equipped with a Scienta-200 hemispherical analyzer. XPS is measured using monochromatized Al Ka with $h v=1486.6 \mathrm{eV}$. All measurements were calibrated by referencing to Fermi level and $\mathrm{Au} 4 \mathrm{f}_{7 / 2}$ peak position of the $\mathrm{Ar}^{+}$ion sputter-clean gold foil. Elemental analysis has been done based on C1s and S2p spectra including normalization for the photoionization cross-section and spectrometer transmission function.

Inverters were fabricated using two substrates with four devices; each substrate was solder to copper conducting plates and wired by hand to pins for easy connection.

Bias stress measurements were carried out inside a nitrogen-filled glove box, using a Keithley 2612AB and home-made MATLAB software, on samples entire processed in air. Stress pulses at $\mathrm{V}_{\mathrm{G}}=-20 \mathrm{~V}$ of $30 \mathrm{~s}, 300 \mathrm{~s}, 1200 \mathrm{~s}, 3600 \mathrm{~s}$, and $3 \mathrm{~h}$ up to approx twelve hours were applied, while transfer characteristics were recorded at the beginning of the whole experiment and at the end of each stress pulse.

Supporting Information. Supporting Information is available from the Wiley Online Library or from the author.

\section{Acknowledgements}

The authors thank the ERC StG 2012-306826 e-GAMES project, the Networking Research Center on Bioengineering, Biomaterials and Nanomedicine (CIBER-BBN), the DGI (Spain) with project BE-WELL CTQ2013-40480-R, and the Generalitat de Catalunya (2014-SGR-17). Research in Sweden was financially supported the Advanced Functional Materials Center at Linköping University, the Önnesjö Foundation, the Knut and Alice Wallenberg Foundation (Power Paper project, scholars), The Swedish Foundation for Strategic Research (SSF, Synergi project). F. G. D. P. thanks Universidad Técnica de Ambato and Secretaría de Educación Superior, Ciencia, Tecnología e Innovación for funding through a doctoral scholarship "Convocatoria abierta 2010”. We also thank Witold Tatkiewicz for his help with the Image-J software. 
[1] C. Reese, Z. Bao, materialstoday 2007, 10, 20.

[2] H. Minemawari, T. Yamada, H. Matsui, J. Tsutsumi, S. Haas, R. Chiba, R. Kumai, T. Hasegawa, Nature 2011, 475, 364.

[3] Y. Diao, L. Shaw, Z. Bao, S. C. B. Mannsfeld, Energy Environ. Sci. 2014, 7, 2145.

[4] Y. Xu, C. Liu, D. Khim, Y.-Y. Noh, Phys. Chem. Chem. Phys. 2014, DOI 10.1039/c4cp02413c.

[5] A. Pierre, M. Sadeghi, M. M. Payne, A. Facchetti, J. E. Anthony, A. C. Arias, Adv. Mater. 2014, 26, 5722.

[6] Y. Diao, B. C.-K. Tee, G. Giri, J. Xu, D. H. Kim, H. A. Becerril, R. M. Stoltenberg, T. H. Lee, G. Xue, S. C. B. Mannsfeld, Z. Bao, Nat. Mater. 2013, 12, 665.

[7] G. Giri, R. Li, D.-M. Smilgies, E. Q. Li, Y. Diao, K. M. Lenn, M. Chiu, D. W. Lin, R. Allen, J. Reinspach, S. C. B. Mannsfeld, S. T. Thoroddsen, P. Clancy, Z. Bao, A. Amassian, Nat. Commun. 2014, 5, DOI 10.1038/ncomms4573.

[8] H. Pan, Y. Li, Y. Wu, P. Liu, B. S. Ong, S. Zhu, G. Xu, J. Am. Chem. Soc. 2007, 129, 4112.

[9] G. Lu, J. Blakesley, S. Himmelberger, P. Pingel, J. Frisch, I. Lieberwirth, I. Salzmann, M. Oehzelt, R. Di Pietro, A. Salleo, N. Koch, D. Neher, Nat. Commun. 2013, 4, 1588.

[10] J. Rivnay, L. H. Jimison, J. E. Northrup, M. F. Toney, R. Noriega, S. Lu, T. J. Marks, A. Facchetti, A. Salleo, Nat. Mater. 2009, 8, 952.

[11] K. Fukuda, Y. Takeda, M. Mizukami, D. Kumaki, S. Tokito, Sci. Rep. 2014, 4, 3947.

[12] K. Kuribara, H. Wang, N. Uchiyama, K. Fukuda, T. Yokota, U. Zschieschang, C. Jaye, D. Fischer, H. Klauk, T. Yamamoto, K. Takimiya, M. Ikeda, H. Kuwabara, T. Sekitani, Y.-L. Loo, T. Someya, Nat. Commun. 2012, 3, 723.

[13] P. a Bobbert, A. Sharma, S. G. J. Mathijssen, M. Kemerink, D. M. de Leeuw, Adv. Mater. 2012, 24, 1146.

[14] C. Piliego, D. Jarzab, G. Gigli, Z. Chen, A. Facchetti, M. A. Loi, Adv. Mater. 2009, 21, 1573.

[15] A. A. Virkar, S. Mannsfeld, Z. Bao, N. Stingelin, Adv. Mater. 2010, 22, 3857.

[16] S. Fabiano, B. Pignataro, Chem. Soc. Rev. 2012, 41, 6859.

[17] S. G. J. Mathijssen, M.-J. Spijkman, A.-M. Andringa, P. A. van Hal, I. McCulloch, M. Kemerink, R. A. J. Janssen, D. M. de Leeuw, Adv. Mater. 2010, 22, 5105.

[18] M. Mas-Torrent, P. Hadley, S. T. Bromley, N. Crivillers, J. Veciana, C. Rovira, Appl. Phys. Lett. 2005, 86, 12110. 
[19] M. Leufgen, O. Rost, C. Gould, G. Schmidt, J. Geurts, L. W. Molenkamp, N. S. Oxtoby, M. Mas-Torrent, N. Crivillers, J. Veciana, C. Rovira, Org. Electron. 2008, 9, 1101.

[20] B. Noda, H. Wada, K. Shibata, T. Yoshino, M. Katsuhara, I. Aoyagi, T. Mori, T. Taguchi, T. Kambayashi, K. Ishikawa, H. Takezoe, Nanotechnology 2007, 18, 424009.

[21] Naraso, J. Nishida, S. Ando, J. Yamaguchi, K. Itaka, H. Koinuma, H. Tada, S. Tokito, Y. Yamashita, J. Am. Chem. Soc. 2005, 127, 10142.

[22] T. Yamada, T. Hasegawa, M. Hiraoka, H. Matsui, Y. Tokura, G. Saito, Appl. Phys. Lett. 2008, 92, 233306.

[23] J. Nagakubo, M. Ashizawa, T. Kawamoto, A. Tanioka, T. Mori, Phys. Chem. Chem. Phys. 2011, 13, 14370.

[24] K. Chen, S. V Stoianov, J. Bangerter, H. D. Robinson, J. Colloid Interface Sci. 2010, 344, 315.

[25] A. S. Dimitrov, K. Nagayama, Langmuir 1996, 12, 1303.

[26] P. Born, S. Blum, A. Munoz, T. Kraus, Langmuir 2011, 27, 8621.

[27] B. R. Hamilton, J. Smith, S. Ogier, M. Heeney, J. E. Anthony, I. Mcculloch, J. Veres, D. D. C. Bradley, T. D. Anthopoulos, Adv. Mater. 2009, 21, 1166.

[28] H. Zhong, J. Smith, S. Rossbauer, A. J. P. White, T. D. Anthopoulos, M. Heeney, Adv. Mater. 2012, 24, 3205.

[29] J. Smith, R. Hamilton, M. Heeney, D. M. de Leeuw, E. Cantatore, J. E. Anthony, I. McCulloch, D. D. C. Bradley, T. D. Anthopoulos, Appl. Phys. Lett. 2008, 93, 253301.

[30] A. Brillante, I. Bilotti, R. G. Della Valle, E. Venuti, M. Mas-Torrent, C. Rovira, Y. Yamashita, Chem. Phys. Lett. 2012, 523, 74.

[31] A. Brillante, I. Bilotti, R. G. Della Valle, E. Venuti, S. Milita, C. Dionigi, F. Borgatti, A. N. Lazar, F. Biscarini, M. Mas-Torrent, N. S. Oxtoby, N. Crivillers, J. Veciana, C. Rovira, M. Leufgen, G. Schmidt, L. W. Molenkamp, CrystEngComm 2008, 10, 1899.

[32] M. Mas-Torrent, C. Rovira, Chem. Rev. 2011, 111, 4833.

[33] R. J. Ashley, in Polym. Permeability SE - 7 (Ed.: J. Comyn), Springer Netherlands, 1985, pp. 269-308.

[34] J. Zaumseil, H. Sirringhaus, Chem. Rev. 2007, 107, 1296.

[35] V. C. Sundar, J. Zaumseil, V. Podzorov, E. Menard, R. L. Willett, T. Someya, M. E. Gershenson, J. A. Rogers, Science (80-. ). 2004, 303, 1644.

[36] D. Venkateshvaran, M. Nikolka, A. Sadhanala, V. Lemaur, M. Zelazny, M. Kepa, M. Hurhangee, A. J. Kronemeijer, V. Pecunia, I. Nasrallah, I. Romanov, K. Broch, I. 
McCulloch, D. Emin, Y. Olivier, J. Cornil, D. Beljonne, H. Sirringhaus, Nature 2014, 515, 384.

[37] X. Zhang, H. Bronstein, A. J. Kronemeijer, J. Smith, Y. Kim, R. J. Kline, L. J. Richter, T. D. Anthopoulos, H. Sirringhaus, K. Song, M. Heeney, W. Zhang, I. McCulloch, D. M. DeLongchamp, Nat Commun 2013, 4.

[38] G. Gelinck, P. Heremans, K. Nomoto, T. D. Anthopoulos, Adv. Mater. 2010, 22, 3778.

[39] D. V Lang, X. Chi, T. Siegrist, a. M. Sergent, a. P. Ramirez, Phys. Rev. Lett. 2004, 93, 86802.

[40] W. Xie, K. Willa, Y. Wu, R. H» usermann, K. Takimiya, B. Batlogg, C. D. Frisbie, Adv. Mater. 2013, 25, 3478.

[41] W. L. Kalb, B. Batlogg, Phys. Rev. B 2010, 81, 035327.

[42] G. Horowitz, M. E. Hajlaoui, R. Hajlaoui, J. Appl. Phys. 2000, 87, 4456.

[43] G. Horowitz, R. Hajlaoui, P. Delannoy, J. Phys. III Fr. 1995, 5, 355.

[44] V. Podzorov, E. Menard, A. Borissov, V. Kiryukhin, J. A. Rogers, M. E. Gershenson, Phys. Rev. Lett. 2004, 93, 86602.

[45] H. Xie, H. Alves, A. F. Morpurgo, Phys. Rev. B 2009, 80, 245305.

[46] N. A. Minder, S. Ono, Z. Chen, A. Facchetti, A. F. Morpurgo, Adv. Mater. 2012, 24, 503.

[47] C. Liu, T. Minari, X. Lu, A. Kumatani, K. Takimiya, K. Tsukagoshi, Adv. Mater. 2011, 23, 523.

[48] S. F. Nelson, Y.-Y. Lin, D. J. Gundlach, T. N. Jackson, Appl. Phys. Lett. 1998, 72, 1854.

[49] T. Sakanoue, H. Sirringhaus, Nat. Mater. 2010, 9, 736.

[50] M. E. Roberts, S. C. B. Mannsfeld, N. Queralto, C. Reese, J. Locklin, W. Knoll, Z. Bao, Proc. Natl. Acad. Sci. 2008, 105, 12134.

[51] M. L. Hammock, O. Knopfmacher, B. D. Naab, J. B.-H. Tok, Z. Bao, ACS Nano 2013, 7, 3970.

[52] M. Ramesh, H.-C. Lin, C.-W. Chu, J. Mater. Chem. 2012, 22, 16506.

[53] N. Lustig, J. Kanicki, J. Appl. Phys. 1989, 65, 3951.

[54] C. Van Berkel, M. J. Powell, Appl. Phys. Lett. 1987, 51, 1094.

[55] W. B. Jackson, J. M. Marshall, M. D. Moyer, Phys. Rev. B 1989, 39, 1164. 
[56] K. S. Karim, A. Nathan, M. Hack, W. I. Milne, Electron Device Lett. IEEE 2004, 25, 188.

[57] A. Stesmans, Y. Wu, Solid State Commun. 1987, 62, 435.

[58] N. Lustig, W. E. Howard, Solid State Commun. 1989, 72, 59. 

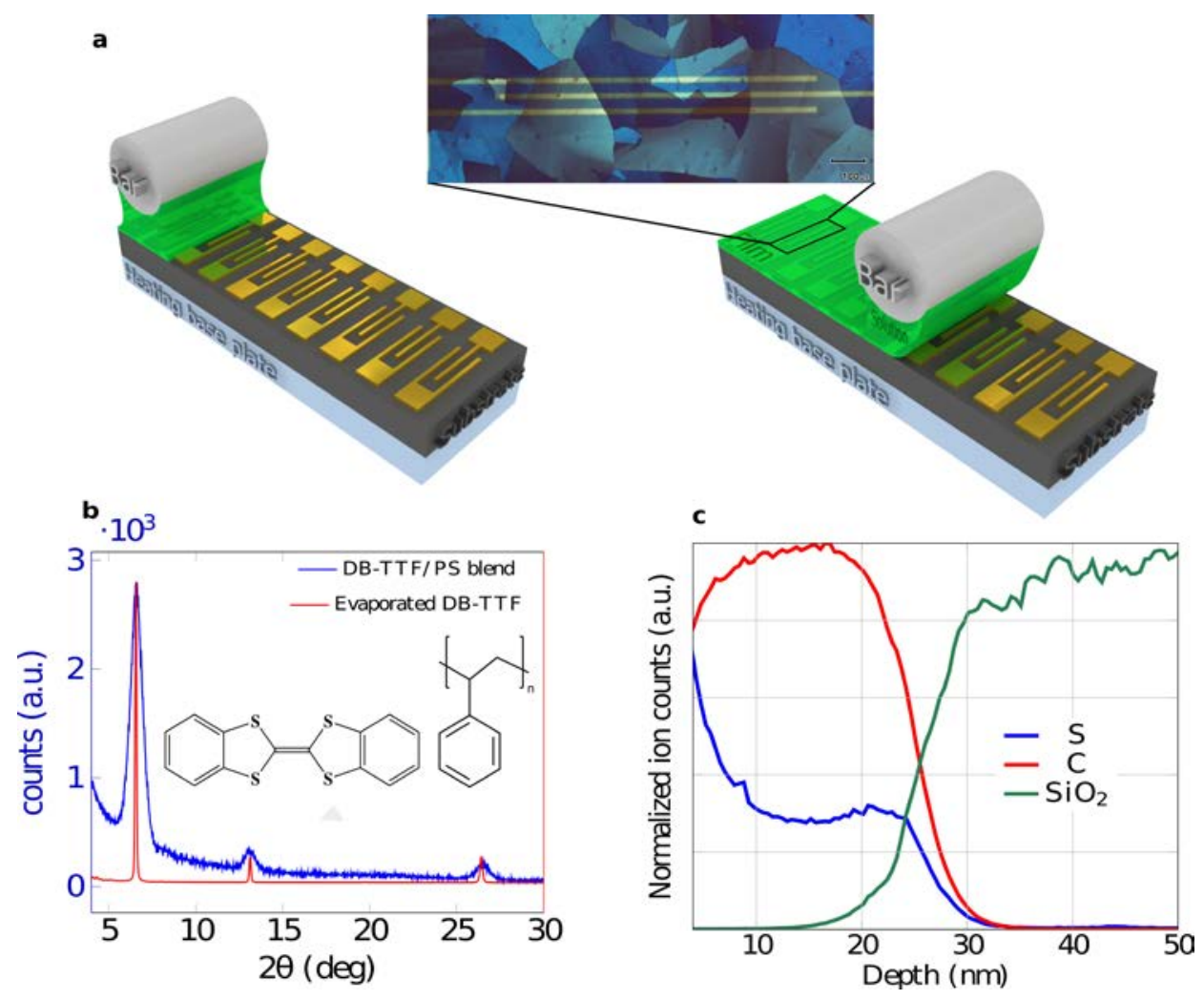

Figure 1. a) Schematic illustration of the BAMs coating technique where the organic semiconductor solution forms first a restricted meniscus between the bar and the substrate which is later on dragged along and a thin-film is formed. Optical polarized microscopy image with crossed polarizer/analyzer setup exhibiting the formation of large crystalline domains. The channel length of the device is $30 \mu \mathrm{m}$. b) XRD diffractogram of thermally evaporated thin films of DB-TTF in comparison with DB-TTF/PS solution-processed films. Inset: molecular structure of DB-TTF and PS. c) ToF-SIMS depth profile of S, C and $\mathrm{SiO}_{2}$ of a typical thin film of DB-TTF/PS starting from the surface and reaching the $\mathrm{SiO}_{2}$ /organic blend interface. 

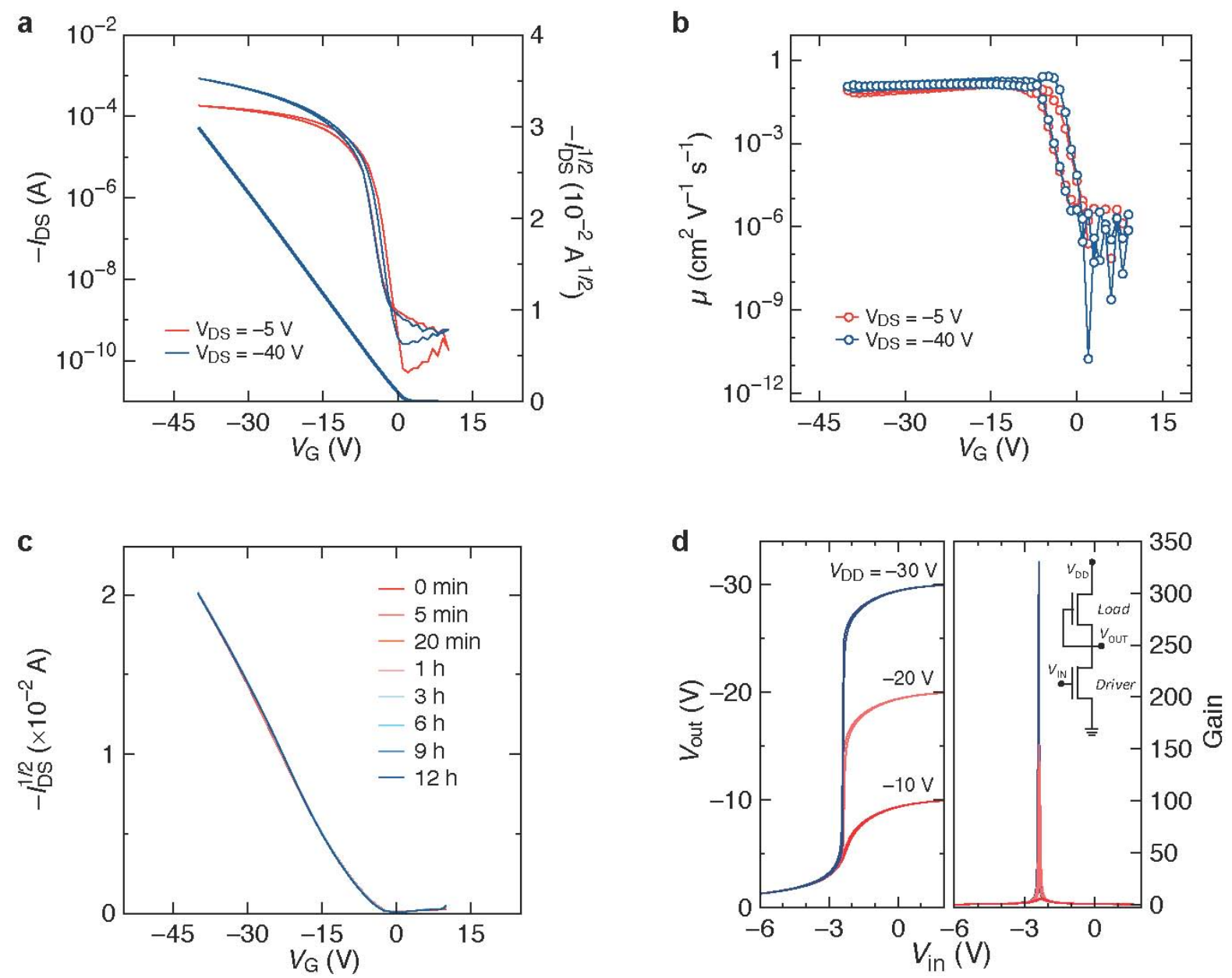

Figure 2. a) Transfer curves and b) mobility profiles for linear and saturation regimes as a function of gate voltage. c) Measured transfer curves $\left(V_{D}=-40 \mathrm{~V}\right)$ after stressing an OFET with a gate bias voltage of $-20 \mathrm{~V}$ for 12 hours. d) Transfer characteristics of an air-exposed depleted load inverter with gain larger than 300 at relatively low supply voltages $\left(V_{D D}=-30 \mathrm{~V}\right)$. 


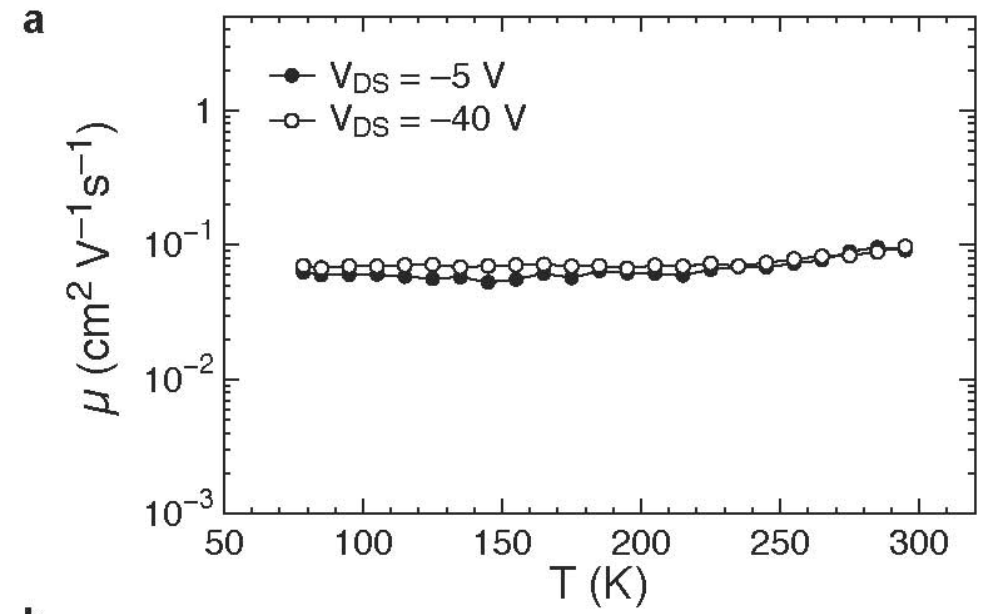

b

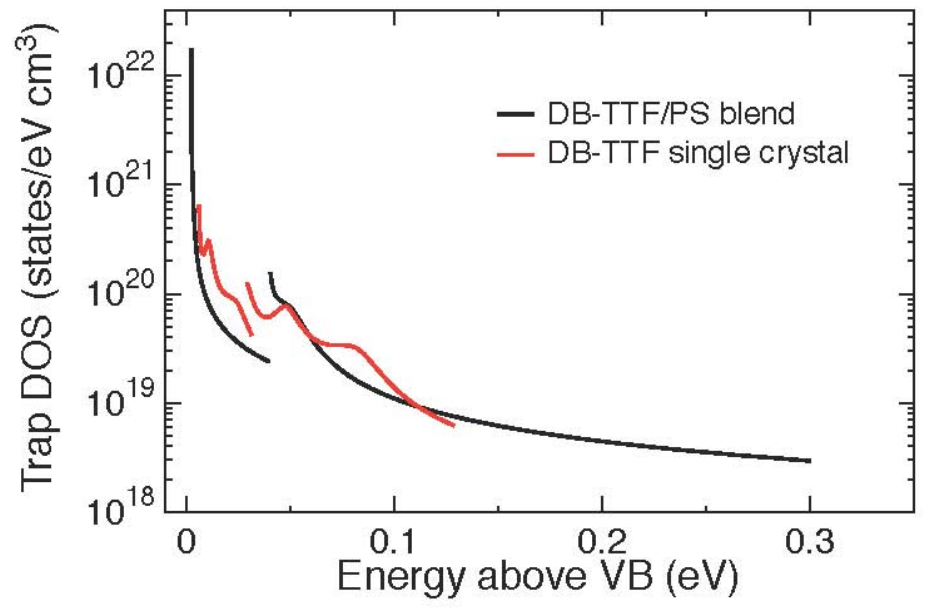

Figure 3. a) Temperature dependence of the mobility for DB-TTF/PS device. b) Trap density of states (trap DOS) of DB-TTF/PS-based FETs plotted against the energy above HOMO level. A comparison with the trap DOS of DB-TTF single crystals from Ref. ${ }^{[19]}$ is presented. 

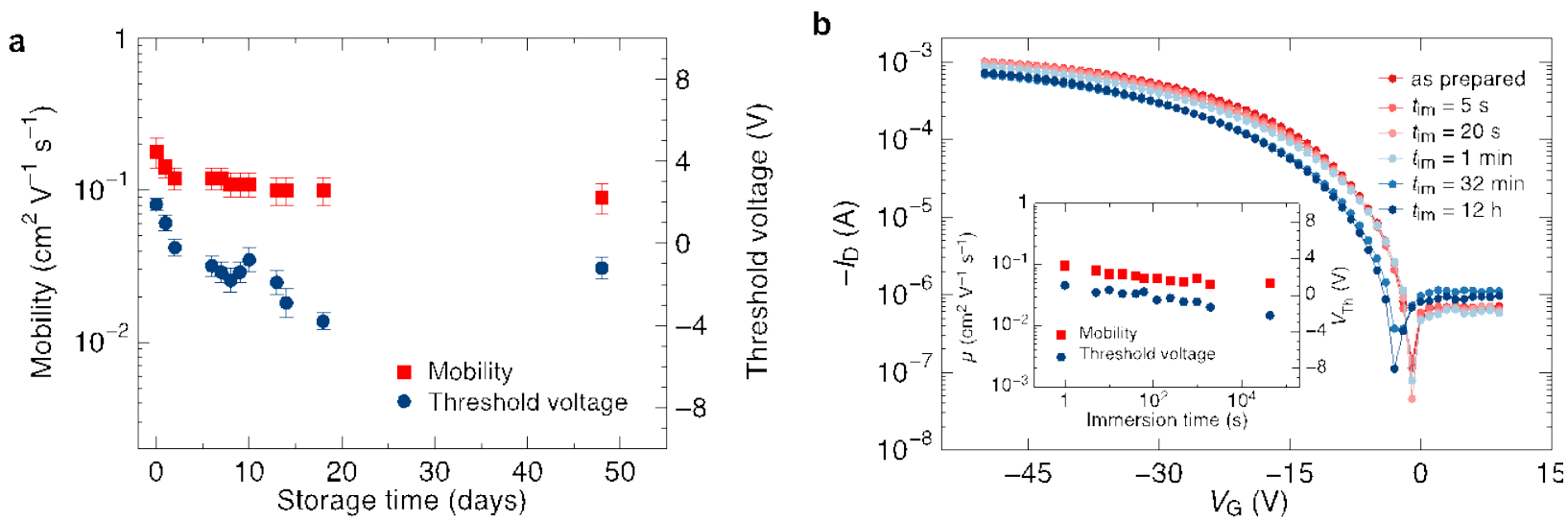

Figure 4. Stability study of FETs based on DB-TTF/PS. a) Shelf stability. Averaged mobility and threshold voltage values of ten devices measured along 48 days. b) Stability in water. Transfer characteristics of a DB-TTF/PS based FET after different immersion times $\left(\mathrm{t}_{\mathrm{im}}=5 \mathrm{~s}\right.$, $20 \mathrm{~s}, 1 \mathrm{~min}, 32 \mathrm{~min}, 12 \mathrm{~h})$ in water. Inset: Mobility extracted in the saturation regime $\left(\mathrm{V}_{\mathrm{D}}=\right.$ $40 \mathrm{~V}$ ) and threshold voltage as a function of water immersion time. 